\title{
3D Characterisation of the Occlusion of Dentine Tubules
}

Harriet Boswell ${ }^{1}$, Arno Merkle ${ }^{2}$, Jeff Gelb ${ }^{2}$, Pete Lander ${ }^{2}$, Jeremy Skepper ${ }^{3}$, Jonathan Earl ${ }^{4}$ and Richard Langford $^{1}$

1. Department of Physics, Cavendish Laboratory, University of Cambridge, Cambridge, UK

2. Xradia Inc. Global Headquarters, Pleasanton, California, USA

3. Department of Physiology, Development and Neuroscience, University of Cambridge, Cambridge, UK

4. GlaxoSmithKline, Consumer Healthcare, Weybridge, Surrey, UK

Dentine tissue has a network of tubules which radiate from the pulp of the tooth. When the tubules are exposed, the movement of intradentinal fluid causes dentine hypersensitivity eliciting pain as a result of hot, cold or chemical stimuli [1]. Prevention of hypersensitivity can be achieved by brushing with desensitising toothpaste with an active ingredient of 45S5 bioactive glass material [2]. 45S5 is a sodium calcium phosphosilicate bioactive glass [3] which occludes the dentine tubules [2] and this research aims to study the nature of the tubule occlusion by $45 \mathrm{~S} 5$ bioactive glass. Correlative analyses using laboratory nano X-ray tomography, focused ion beam (FIB) slice and view and energy dispersive X-ray spectroscopy (EDS) give physical and chemical information about the system which can be reconstructed in three dimensions.

Dentine discs cut parallel to the dentine-enamel junction were supplied by GlaxoSmithKline and were prepared into $35 \mu \mathrm{m}$ by $35 \mu \mathrm{m}$ pillars and etched using $6 \%$ citric acid for 30 seconds. Initial analysis of the dentine sample was carried out using laboratory nanoscale X-ray microscopy (Xradia UltraXRML200) in order to image the pillars in three dimensions non-destructively. This approach allows quantification of the dentine microstructure, to complement the subsequent destructive electron microscopic investigations. Figure 1 shows dentine tubules ranging in size from 1 to $5 \mu \mathrm{m}$ in diameter and varying depths depending on the amount of branching of each tubule.

A separate dentine pillar was immersed in a solution of $100 \mathrm{ml}$ artificial saliva and $50 \mathrm{mg} 45 \mathrm{~S} 5$ and left for 40 minutes at $37^{\circ} \mathrm{C}$. Subsequently FIB slice and view was carried out on the Zeiss CrossBeam $1540 \mathrm{XB}$ using a milling condition of $30 \mathrm{kV}: 500 \mathrm{pA}$ and imaging at $2 \mathrm{kV}$ using the electron column. Reconstructing the secondary electron (SE) images (Figure 2a) shows that the 45S5 provides high percentage occlusion within the tubules. The main constituent of dentine is hydroxyapatite which has a $\mathrm{Ca} / \mathrm{P}$ ratio of 1.67. EDS was carried out at $20 \mathrm{kV}$. Figure $2 \mathrm{~b}$ shows a $\mathrm{Ca} / \mathrm{P}$ EDS map of one of the FIB slices demonstrating that the dentine matrix has a similar $\mathrm{Ca} / \mathrm{P}$ ratio value to that of hydroxyapatite but the ratio increases at the sites of occlusion. This is in part due to the irregular shape and size of the tubules (as illustrated in the UltraXRM preliminary experiment (Figure 1)) and the large interaction volume from which the X-ray signals derive from. A significant limitation of this technique is from the difference in interaction depths associated with the EDS and SE signals.

These initial results have highlighted some of the problems of analysing the dentine / bioactive glass system - mainly the irregular size and shape of the tubules and non-uniform nature of the occluding material. Using a marker system e.g. a substituted 45S5 bioactive glass, it is hoped that EDS will be able to better map the occlusion so the percentage filling can be calculated. Furthermore, using nano Xray tomography, FIB slice and view and EDS on the same sample will enable analysis of the system over a range of resolutions to better understand the nature of the occlusion. 
References:

[1] M.A. Brännström in "Sensory Mechanism in Dentin", ed. D.J. Anderson, (1963, Pergammon Press, Oxford) p. 73-79.

[2] D.G. Gillam et al, Journal of Oral Rehabilitation, 29 (2002) p. 305-313.

[3] L.L. Hench, J. Mater Sci: Mater Med, 17 (2006) p. 967-978.

[4] The authors acknowledge funding from the Biotechnology and Biological Sciences Research

Council (BBSRC) and GlaxoSmithKline.
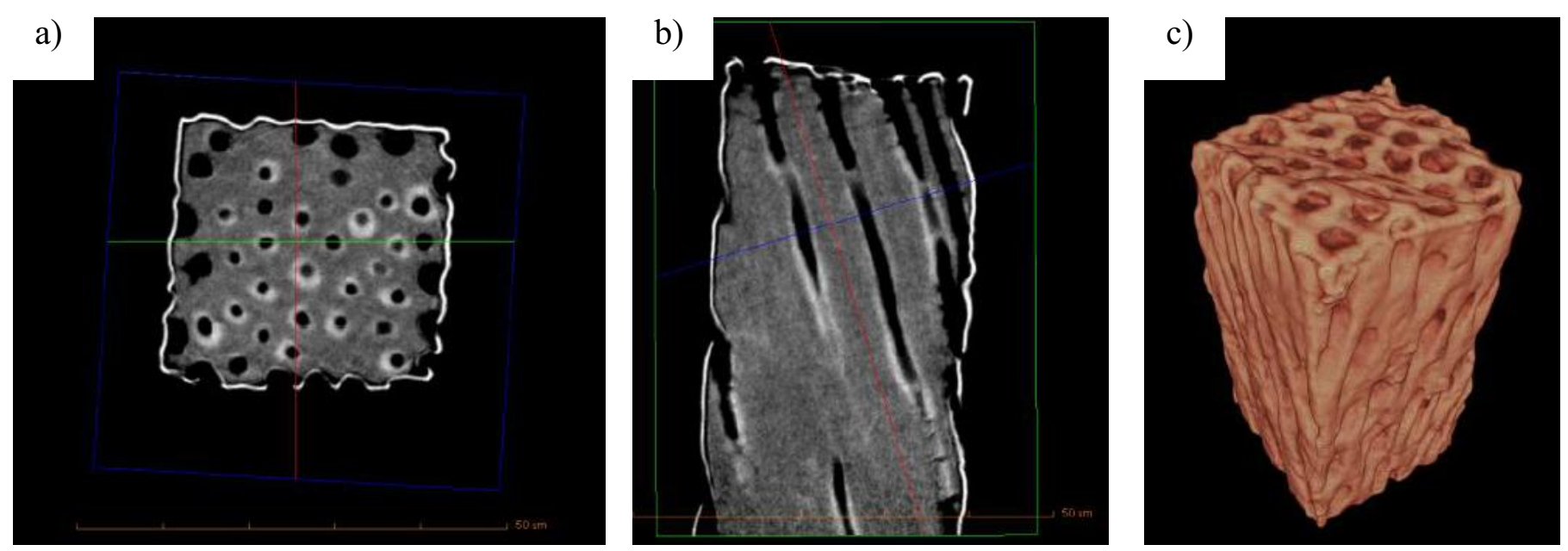

Figure 1. Nano X-ray tomography $(8 \mathrm{keV})$ reconstructed images of a dentine pillar etched with citric acid a) virtual 2D slice top down view, b) virtual 2D slice side on view and c) 3D volume rendering (pillar width $\sim 35 \mu \mathrm{m}$ ).

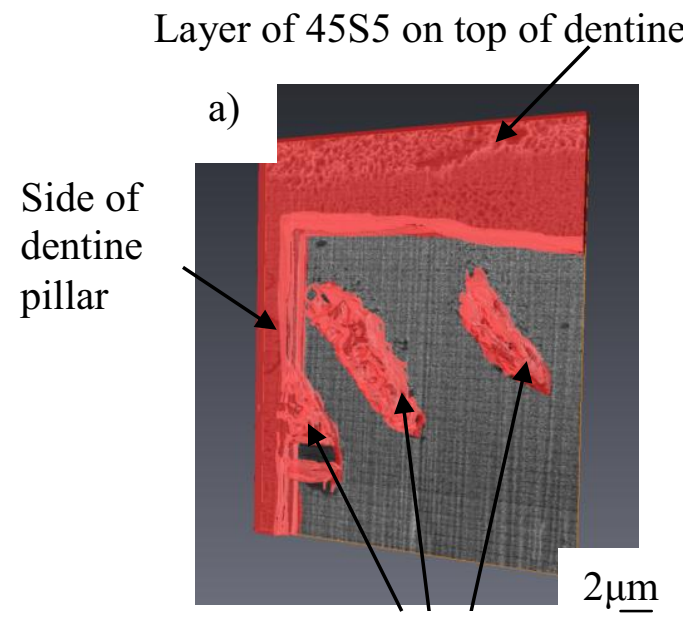

Occlusion of dentine tubures

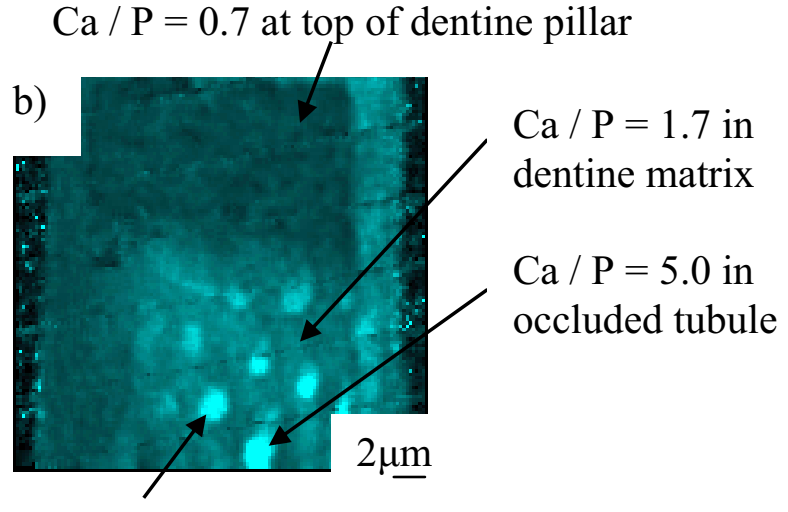

$\mathrm{Ca} / \mathrm{P}=3.0$ in

occluded tubule

Figure 2. a) FIB slice and view SE image 3D reconstruction of $45 \mathrm{~S} 5$ (shown in red) occluding the dentine tubules and b) EDS map of one slice showing the $\mathrm{Ca} / \mathrm{P}$ ratios at different points in the sample. 